\title{
BMJ Open Interventions designed to increase the uptake of lung cancer screening and implications for priority populations: a scoping review protocol
}

\author{
Ambreen Sayani (D) ,1,2 Muhanad Ahmed Ali, ${ }^{1}$ Ann Marie Corrado, ${ }^{1,3}$ \\ Carolyn Ziegler, ${ }^{4}$ Alex Sadler, ${ }^{1}$ Christina Williams, ${ }^{1}$ Aisha Lofters ${ }^{1,2,3,5,6,7,8}$
}

To cite: Sayani A, Ali MA, Corrado AM, et al. Interventions designed to increase the uptake of lung cancer screening and implications for priority populations: a scoping review protocol. BMJ Open 2021;11:e050056. doi:10.1136/ bmjopen-2021-050056

- Prepublication history and additional supplemental material for this paper are available online. To view these files, please visit the journal online (http://dx.doi.org/10.1136/ bmjopen-2021-050056).

Received 09 February 2021 Accepted 12 July 2021

Check for updates

(C) Author(s) (or their employer(s)) 2021. Re-use permitted under CC BY-NC. No commercial re-use. See rights and permissions. Published by BMJ.

For numbered affiliations see end of article.

Correspondence to

Dr Ambreen Sayani;

ambreen.sayani@wchospital.ca

\section{ABSTRACT}

Background When designing any health intervention, it is important to respond to the unequal determinants of health by prioritising the allocation of resources and tailoring interventions based on the disproportionate burden of illness. This approach, called the targeting of priority populations, can prevent a widening of health inequities, particularly those inequities which can be further widened by differences in the uptake of an intervention. The objective of this scoping review is to describe intervention(s) designed to increase the uptake of lung cancer screening, including the health impact on priority populations and to describe knowledge and implementation gaps to inform the design of equitable lung cancer screening.

Methods We will conduct a scoping review following the methodological framework developed by Arksey and 0'Malley. We will conduct comprehensive searches for lung cancer screening promotion interventions in Ovid Medline, Embase, the Cochrane Library, Cumulative Index to Nursing \& Allied Health (CINAHL) and Scopus. We will include published English language peer-reviewed and grey literature published between January 2000 and 2020 that describe an intervention designed to increase the uptake of low-dose CT (LDCT) lung cancer screening in the Organization for Economic Cooperation and Development countries. Articles not in English or not describing LDCT will be excluded. Three authors will review retrieved literature in three steps: title, abstract and then full text. Three additional authors will review discrepancies. Authors will extract data from full-text papers into a chart adapted from the Template for Intervention Description and Republication checklist, the Consolidated Standards of Reporting Trials and a Health Equity Impact Assessment tool. Findings will be presented using a narrative synthesis. Ethics and dissemination The knowledge synthesised will be used to inform the equitable design of lung cancer screening and disseminated through conferences, publications and shared with relevant partners. The study does not require research ethics approval as literature is available online.

\section{BACKGROUND}

Lung cancer is the most commonly diagnosed cancer in Canada and is responsible
Strengths and limitations of this study

- This scoping review will provide an overview of existing lung cancer screening interventions designed to increase the uptake of screening participation with low-dose CT.

- Lung cancer screening with low-dose CT is relatively new; therefore, we anticipate retrieving a limited numbers of publications.

- We will only include articles in the English language and may miss knowledge about interventions that have been published in other languages.

- We will analyse interventions using a Health Equity Impact Assessment tool to illuminate impact of the intervention on priority populations.

- We will provide a timely synthesis of knowledge and implementation gaps to inform equitable access to lung cancer screening.

for a quarter of all cancer-related deaths in the country. ${ }^{1}$ Lung cancer associated mortality is typically linked to advanced stage tumours and therefore screening for earlystage and curable lung cancer with the use of low-dose CT (LDCT) is an important way to potentially reduce mortality. ${ }^{2}$ Organised lung cancer screening through public health programmes is currently a health system priority in Canada ${ }^{4}$ and is being initiated in the provinces of Ontario and British Columbia. ${ }^{56}$

Lung cancer screening is offered to individuals who are considered high risk based on age (between 55 and 74 years) and pack-year smoking history (30 pack years, with pack year defined as the (average number of cigarette packs smoked daily) $\times$ (number of years smoking) ). ${ }^{7}$ Significantly, the unequal distribution of the social determinants of health which underpin smoking behaviour are clustered together with differences in the ability to access healthcare ${ }^{8}$ leading to inequities 


\section{Box 1 Priority population approach}

According to Ontario Public Health Standards populations can be prioritised by:

1. Taking specific action on reducing disease burden for disadvantaged populations that is proportional to their specific needs.

2. Designing interventions that are proportional to the burden of disease.

3. Allocating resources and services based on the anticipated need of the population.

in lung cancer risk and mortality. ${ }^{1}$ Conditions of social disadvantage lead to economic, structural and geographical barriers to healthcare for certain population groups. ${ }^{9}$ As a result, individuals living with greater degrees of social disadvantage such as low income are more likely to be smoking, ${ }^{10}$ face a higher risk of developing lung cancer, ${ }^{11}$ are less likely to participate in lung cancer screening ${ }^{8} 12$ and have a higher rate of mortality due to lung cancer. ${ }^{1}$

In Canada, historical injustice and discrimination against Indigenous populations, a shortage of specialised services in rural and remote geographical areas and stigma associated with smoking and poverty create specific barriers to lung cancer screening participation. ${ }^{813}$ These populations, described as priority populations, require action at the policy and systems level to reduce lung cancer risk ${ }^{14}$ as well as specific interventions designed to increase access to and uptake of lung cancer screening to promote early detection of lung cancer. The proactive design of interventions to increase the uptake of lung cancer screening among populations experiencing the greatest health inequities is described as a priority populations approach (see box 1). Such an approach, places an emphasis on the reallocation of resources and services based on expected need, higher burden of illness, and barriers to care in order to prevent a widening of health inequities which are rooted in social inequities. ${ }^{15}$

Interventions designed to improve the uptake of lung cancer screening are more likely to promote equitable health outcomes if a priority population approach is applied. However, little is currently known about the range and nature of interventions which have been designed to enhance the uptake of lung cancer screening and what impact they have on priority populations (intended/ unintended or positive/negative). This knowledge is important to illuminate potential gaps in health service design and prevent a further widening of health inequities as a result of lung cancer screening interventions.

\section{METHODS}

We are following the research approach that conforms with the Preferred Reporting Items for Systematic Reviews and Meta-Analysis extension for Scoping Reviews. ${ }^{16}$ Methodologically, scoping reviews can be used to assess literature for the breadth and depth of knowledge in a given field. Specifically, scoping reviews can be used to develop new understandings by searching, selecting and synthesising key concepts, ideologies and gaps in an area without emphasis on the quality of published literature. ${ }^{17} 18$ Publishing this protocol is a critical step in documenting our scoping review plan. Specifically, this paper will use the methodological framework developed by Arksey and $\mathrm{O}^{\prime}$ Malley ${ }^{17}$ to guide the six stages of our scoping study design as outlined below:

\section{Stage 1: identification of the research question}

The objective of this scoping study is to provide an overview of existing interventions designed to increase the uptake of lung cancer screening and the potential impact on priority populations. This will illuminate implementation gaps for priority populations in order to inform the design and delivery of equitable lung cancer screening. Our scoping review will address the following research questions:

1. What interventions have been implemented to increase the uptake of lung cancer screening in the Organization for Economic Cooperation and Development (OECD) countries (including study design, population, nature of intervention and outcome measures), and were these interventions effective?

2. What are the health impacts of the intervention (intended/unintended and positive/negative) on priority populations?

3. What knowledge and implementation gaps can we identify to inform the equitable design of lung cancer screening?

\section{Stage 2: identifying relevant studies}

All named authors have participated in an iterative process to develop the initial search strategy beginning in July 2020.This includes identifying key terms, inclusion and exclusion criteria and relevant databases. The following databases will be used to conduct the peerreviewed literature search: Ovid Medline, Embase, the Cochrane Library, CINAHL and Scopus. The search strategies, adapted for each database, will use a comprehensive combination of subject headings and keywords for the concepts lung cancer screening and health promotion interventions. The detailed Medline search strategy can be found in (online supplemental file 1). Additionally, grey literature documents related to lung cancer screening interventions and pilot programmes (ie, policy documents and reports) will be identified using government and institutional websites. The search strategy has been peer reviewed by another librarian external to the study team using the Canadian Agency for Drugs and Technologies in Health Peer Review of Electronic Search Strategies Checklist. ${ }^{19}$

\section{Stage 3: study selection}

We will select articles published between January 2000 and 2020. Clinical trials evaluating LDCT for lung cancer screening to detect early-stage tumours began in the early $2000 \mathrm{~s}^{20}$ and therefore we do not expect 


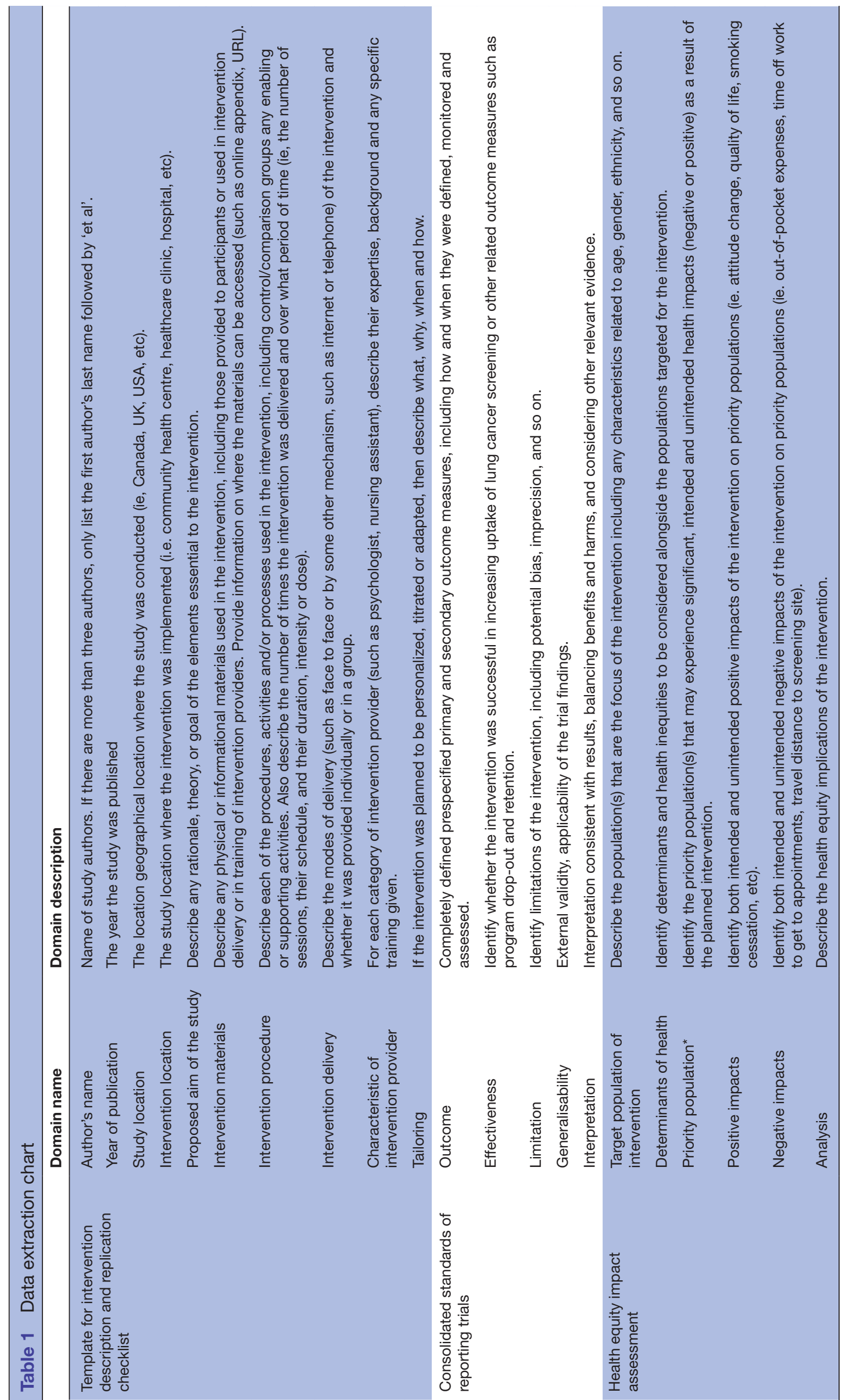

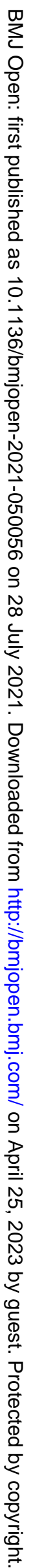


to find any relevant literature prior to the year 2000. We will include all published literature in the English language that focuses on lung cancer screening with LDCT that describes an intervention designed to increase the uptake of lung cancer screening. We will include interventions that occurred in jurisdictions similar to the Canadian context that is, jurisdictions that are members, or that are in countries that are members, of the OECD. We will include peer-reviewed publications, abstracts, dissertations; or grey literature, such as government documents, conference proceedings and institutional repositories. Three researchers (MA, ALS, CW) will independently screen the titles and abstracts for each citation to identify articles eligible for full text review. Discrepancies will be discussed and resolved through consultation with the larger team (AS, AMC, AL). All eligible publications will proceed to full-text review and data extraction.

\section{Stage 4: charting the data}

Data from included publications will be extracted and charted in a template (table 1) adapted from the Template for Intervention Description and Republication checklist, ${ }^{21}$ the Consolidated Standards of Reporting Trials, ${ }^{22}$ and the Ontario Ministry of Health's Health Equity Impact Assessment (HEIA) tool. ${ }^{23}$ As a first step, data will be descriptively charted from the literature, including the target population of the intervention, characteristics of the intervention (including the integration of smoking cessation programmes if any) and reported effectiveness measures. Subsequently, the study team will use the HEIA tool to chart intended/unintended and positive/negative impacts of each intervention on priority populations. Team members will independently chart the first three articles and review as a larger team for discrepancies. The remaining articles will be distributed for data charting and the full team will meet for regular scheduled review to guide the process. Any remaining discrepancies will be resolved by team members (AS and AL).

\section{Stage 5: collating, summarising and reporting the results}

The findings from the scoping review will be presented using a narrative synthesis. Specifically, we will summarise and synthesise the data extracted from the studies and illuminate any knowledge and implementation gaps for priority populations. This knowledge can promote the equitable design of future lung cancer screening interventions given the disproportionate burden of lung cancer in populations experiencing varying degrees of social disadvantage. We anticipate concluding our study by December 2021.

\section{Patient and public involvement}

Our study design and research questions have been informed through formal and informal consultations with patients, community partners and decision-makers at the federal and provincial level. We will consult stakeholders involved in the implementation of lung cancer screening in Canada in the analysis of our findings. We will involve patients in the identification of appropriate knowledge dissemination tools.

\section{Ethics and dissemination}

No ethics approval was needed for this study as data will be collected from publicly available literature. Once published, the data will be shared with relevant stakeholders and community members at large, including dissemination through peer-reviewed journals and conference presentations.

\section{Author affiliations}

${ }^{1}$ Women's College Research Institute, Women's College Hospital, Toronto, Ontario, Canada

${ }^{2}$ MAP - Centre for Urban Health Solutions, Unity Health Toronto, Toronto, Ontario, Canada

${ }^{3}$ Peter Gilgan Centre for Women's Cancers, Women's College Hospital, Toronto, Ontario, Canada

${ }^{4}$ Library Services, Unity Health Toronto, Toronto, Ontario, Canada

${ }^{5}$ ICES, Toronto, Ontario, Canada

${ }^{6}$ Department of Family and Community Medicine, University of Toronto, Toronto, Ontario, Canada

${ }^{7}$ Department of Family Medicine, Women's College Hospital, Toronto, Ontario, Canada

${ }^{8}$ Dalla Lana School of Public Health, University of Toronto, Toronto, Ontario, Canada

Twitter Ambreen Sayani @SayaniAmbreen

Acknowledgements AS is supported by a Postdoctoral Award in patient-oriented research from the Canadian Institutes of Health Research. AL and AMC are supported by the Peter Gilgan Centre for Women's Cancers at Women's College Hospital, in partnership with the Canadian Cancer Society.

Contributors AS, AMC, CZ and AL contributed to the study conception and design. The first draft of the manuscript was written by AS and MAA. ALS, CW, AMC, CZ and $A L$ commented on previous versions of the manuscript. All authors read and approved the final manuscript.

Funding This study is supported by grant \#705592 from the Canadian Institutes of Health Research and Canadian Cancer Society.

Disclaimer The funding body had no role in the design of the study and writing of the manuscript.

Competing interests None declared.

Patient consent for publication Not required.

Provenance and peer review Not commissioned; externally peer reviewed.

Supplemental material This content has been supplied by the author(s). It has not been vetted by BMJ Publishing Group Limited (BMJ) and may not have been peer-reviewed. Any opinions or recommendations discussed are solely those of the author(s) and are not endorsed by BMJ. BMJ disclaims all liability and responsibility arising from any reliance placed on the content. Where the content includes any translated material, BMJ does not warrant the accuracy and reliability of the translations (including but not limited to local regulations, clinical guidelines, terminology, drug names and drug dosages), and is not responsible for any error and/or omissions arising from translation and adaptation or otherwise.

Open access This is an open access article distributed in accordance with the Creative Commons Attribution Non Commercial (CC BY-NC 4.0) license, which permits others to distribute, remix, adapt, build upon this work non-commercially, and license their derivative works on different terms, provided the original work is properly cited, appropriate credit is given, any changes made indicated, and the use is non-commercial. See: http://creativecommons.org/licenses/by-nc/4.0/.

ORCID iD

Ambreen Sayani http://orcid.org/0000-0001-5391-7769 


\section{REFERENCES}

1 Canadian Cancer Statistics Advisory Committee, Canadian Cancer Society. Canadian cancer statistics: a 2020 special report on lung cancer, 2020. Available: https://www.cancer.ca/ /media/cancer.ca/ CW/cancer\%20information/cancer\%20101/Canadian\%20cancer\% 20statistics/Canadian-cancer-statistics-2020_special-report_EN.pdf? la=en [Accessed 20 Nov 2020].

2 de Koning HJ, van der Aalst CM, de Jong PA, et al. Reduced lungcancer mortality with volume CT screening in a randomized trial. $N$ Engl J Med 2020;382:503-13.

3 National Lung Screening Trial Research Team, Aberle DR, Adams AM, et al. Reduced lung-cancer mortality with low-dose computed tomographic screening. N Engl J Med 2011;365:395-409.

4 Canadian Partnership Against Cancer. The Canadian strategy for cancer control: 2019-2029, 2019. Available: https://www.partners hipagainstcancer.ca/cancer-strategy/

5 Brtish Columbia Cancer Agency. The BC lung screen trial [internet]. Available: http://www.bccancer.bc.ca/our-research/participate/lunghealth [Accessed 20 Nov 2020].

6 Cancer Care Ontario. Lung cancer screening pilot for people at high risk [Internet], 2017. Available: https://www.cancercareontario.ca/ en/guidelines-advice/cancer-continuum/screening/lung-cancerscreening-pilot-people-at-high-risk [Accessed 14 Sep 2018].

7 Canadian Task Force on Preventive Health Care. Lung cancer screening guidelines [Internet]. Available: https://canadiantaskforce. $\mathrm{ca} /$ new-lung-cancer-screening-guideline/ [Accessed 22 Oct 2019].

8 Sayani A, Vahabi M, O'Brien MA, et al. Advancing health equity in cancer care: the lived experiences of poverty and access to lung cancer screening. PLoS One 2021;16:e0251264.

9 Sayani A. Health equity in National cancer control plans: an analysis of the Ontario cancer plan. Int J Health Policy Manag 2019;8:550-6.

10 Jarvis MJ, Wardle J. Social patterning of individual health behaviours: the case of cigarette smoking. In: Marmot M, Wilkinson RG, eds. Social Determinants of Health [Internet]. Oxford: Oxford University Press, 2005. http://discovery.ucl.ac.uk/94245/

11 Mitra D, Shaw A, Tjepkema M, et al. Social determinants of lung cancer incidence in Canada: a 13-year prospective study. Health Rep 2015;26:11.
12 Ali N, Lifford KJ, Carter B, et al. Barriers to uptake among high-risk individuals declining participation in lung cancer screening: a mixed methods analysis of the UK lung cancer screening (UKLS) trial. BMJ Open 2015:5:e008254.

13 Canadian Partnership Against Cancer. Lung cancer and equity: a focus on income and geography, 2020. Available: https://www.part nershipagainstcancer.ca/topics/lung-cancer-equity/

14 Sayani A, Vahabi M, O'Brien MA, et al. Perspectives of family physicians towards access to lung cancer screening for individuals living with low income - a qualitative study. BMC Fam Pract 2021;22:10

15 Minister of Health and Long-Term Care. Ontario public health standards, 2008. Available: https://www.health.gov.on.ca/en/pro/ programs/publichealth/oph_standards/

16 Tricco AC, Lillie E, Zarin W, et al. PRISMA extension for scoping reviews (PRISMA-SCR): checklist and explanation. Ann Intern Med 2018;169:467-73.

17 Arksey H, O'Malley L. Scoping studies: towards a methodological framework. Int J Soc Res Methodol 2005;8:19-32.

18 Levac D, Colquhoun H, O'Brien KK. Scoping studies: advancing the methodology. Implement Sci 2010;5:69.

19 McGowan J, Sampson M, Salzwedel DM, et al. PRESS Peer Review of Electronic Search Strategies: 2015 Guideline Statement. J Clin Epidemiol 2016;75:40-6.

20 Sharma D, Newman TG, Aronow WS. Lung cancer screening: history, current perspectives, and future directions. Arch Med Sci 2015;11:1033-43.

21 Hoffmann TC, Glasziou PP, Boutron I, et al. Better reporting of interventions: template for intervention description and replication (TIDieR) checklist and guide. BMJ 2014;348:g1687.

22 Turner L, Shamseer L, Altman DG, et al. Consolidated standards of reporting trials (consort) and the completeness of reporting of randomised controlled trials (RCTs) published in medical journals. Cochrane Database Syst Rev 2012;2013.

23 Ministry of Health and Long Term Care. Health Equity Impact Assessment (HEIA) tool [Internet], 2012. Available: http://www.health. gov.on.ca/en/pro/programs/heia/docs/template.pdf [Accessed 20 Nov 2020]. 